\title{
Comparison of HER-2 Amplification with Clinicopathological and Prognostic Parameters in Metastatic Gastric Carcinomas
}

\author{
Melin Gecer* \\ Bezmialem Foundation University Hospital Department of Pathology \\ Adnan menderes bulvarı, Vatan street Fatih-Istanbul,Turkey \\ Seval Turna \\ Bezmialem Foundation University Hospital Department of Pathology \\ Adnan menderes bulvarı, Vatan street Fatih-Istanbul,Turkey \\ Zuhal Gucin \\ Bezmialem Foundation University Hospital Department of Pathology \\ Adnan menderes bulvarı, Vatan street Fatih-Istanbul,Turkey
}

\begin{abstract}
Introduction: HER2 overexpression is present in 7 to $34 \%$ of gastric carcinomas. Several studies have demonstrated associations between HER2 overexpression and clinicopathological variables, including tumor depth, lymph node metastasis, and intestinal morphology. HER2 overexpression has been linked to shorter survival.
\end{abstract}

Aim: In this study, we aimed to determine the frequency of HER2 overexpression in patients with metastatic gastric carcinoma referred to our clinic, to assess HER2 expression using immunohistochemistry (IHC) or silver in situ hybridization (SISH) and to demonstrate potential associations between HER2 expression and histopathological parameters.

Materials and Methods: In this study, we assessed archival samples from 120 patients diagnosed with metastatic gastric carcinoma between 2015 and 2019 and tested for HER2 status. Samples had been obtained by endoscopic biopsy in 84 patients and gastric resection in 36 patients, whereas 36 patients were diagnosed in other health facilities and were referred to our department for consulting. Hematoxylin-eosin stained preparations were reassessed, and diagnoses were confirmed based on World Health Organization (WHO), and Lauren classifications and HER2 results were compared to previous results.

HER2 status was assessed by immunohistochemistry (IHC) or silver in situ hybridization (SISH) in available paraffin-embedded tumor sections. Associations between HER2 expression levels and age, sex, tumor location, size and histological type of the tumor, lymphovascular, perineural and perinodal invasion and perinodal invasion, lymph node metastasis, and site of metastasis.

Results: $84(70 \%)$ out of 120 patients with metastatic gastric carcinoma were male, and $36(30 \%)$ were female, and the mean age was 60.7 years (age range: $21-90$ years). 84 patients had undergone an endoscopic biopsy, and 36 patients had undergone gastric resection, whereas 39 patients were diagnosed in other health facilities and were referred to our department for consulting.

In total $31(25.8 \%)$ out of 120 subjects tested positive for HER2 overexpression

Comparisons of histological patterns, according to Lauren's classification, indicated that intestinal type was predominant in both groups, and none of the diffuse gastric carcinomas was HER2 positive. A statistically significant intergroup difference was found with respect to the prevalence of diffuse gastric carcinoma $(p=0.03)$.

Conclusion: In our study, HER-2 gene amplification $(25.8 \%)$ is compatible with the literature. Her2 positive tumors were mostly located proximally and were not observed in diffuse type. In this respect, they were found statistically significant.

Keywords:Her2,SISH,Gastric Carcinoma

Original Article:

DOI: $10.7176 / \mathrm{JHMN} / 79-07$

Publication date:August $31^{\text {st }} 2020$

\section{INTRODUCTION}

Gastric Carcinoma is the fourth most common malignant tumor worldwide after lung, breast, colorectal and prostate cancers and ranks fifth among the most common cancers in Turkey. Gastric Carcinoma is the third common cause of cancer deaths worldwide and the fourth common cause of cancer deaths in our country $(1,2)$. Despite diagnostic and therapeutic advances in gastric carcinoma, the prognosis is poor, particularly in patients with advanced disease; targeted therapies have been developed to improve the response rate in these patients. HER2 gene is a potential target in gastric carcinoma, and HER2 gene amplification is under investigation in various tumors. 
The proto-oncogene HER2 located on the long arm of the seventeenth chromosome encodes the erbB2 receptor protein in the cell membrane. The erbB2 receptor protein is one of the epidermal growth factor receptors, and once activated, it affects cell differentiation, apoptosis, cell adhesion, migration, and growth functions through tyrosine kinase. The erbB2 receptor protein is within the signaling pathways that take part in cell proliferation and is a driver in various cancer types. An increase in the number of HER2 receptors overexpression of the receptor on the plasma membrane compared to normal cells, is investigated with immunohistochemistry techniques in pathology practice $(3,4)$. An increased copy number of the gene encoding the receptor protein in the nucleus, namely gene amplification is investigated with in situ hybridization (ISH) techniques including fluorescence ISH (FISH), chromogenic ISH (CISH) and silver- enhanced ISH (SISH) (5).

The determination of HER2 status has become a standard practice in breast cancers, as it can provide predictive and prognostic information. HER2 receptor blockade in cancer cells in HER2-positive breast tumors may help to inhibit these signaling pathways and prevent tumor cell proliferation. Trastuzumab, an anti-HER2 receptor antibody, has been widely used in HER2 overexpressing breast cancers. HER2-overexpression and amplification analyses have been starte in advanced gastric cancers after the determination of their predictive value $(6,7)$

HER2 overexpression is present in 7 to $34 \%$ of gastric carcinomas. Several studies have demonstrated associations between HER2 overexpression and clinicopathological variables, including tumor depth, lymph node metastasis, and intestinal morphology. HER2 overexpression has been linked to shorter survival $(6,8,9,10,11)$.

In this study, we aimed to determine the frequency of HER 2 overexpression in patients with metastatic gastric carcinoma referred to our clinic, to assess HER2 expression using immunohistochemistry (IHC) or silver in situ hybridization (SISH) and to demonstrate potential associations between HER2 expression and histopathological parameters.

\section{MATERIALS AND METHODS}

In this study, we assessed archival samples from 120 patients diagnosed with metastatic gastric carcinoma between 2015 and 2019 and tested for HER2 status. Samples had been obtained by endoscopic biopsy in 84 patients and gastric resection in 36 patients, whereas 36 patients were diagnosed in other health facilities and were referred to our department for consulting. Hematoxylin-eosin stained preparations were reassessed, and diagnoses were confirmed based on World Health Organization (WHO), and Lauren classifications and HER2 results were compared to previous results.

HER2 status was assessed by immunohistochemistry (IHC) or silver in situ hybridization (SISH) in available paraffin-embedded tumor sections. Associations between HER2 expression levels and age, sex, tumor location, size and histological type of the tumor, lymphovascular, perineural and perinodal invasion and perinodal invasion, lymph node metastasis, and site of metastasis.

\section{Methods for Evaluating HER2 Status}

HER2 immunohistochemistry (IHC) has been performed in all cases, as requested by their primary oncology clinic. Anti-HER2/neu (4B5) rabbit monoclonal antibody (Ventana) was used for IHC staining.

A scoring system proposed by Hoffmann et al. was used for the assessment of HER2 status in gastric carcinoma samples $(3,5)$. This is a 0 to 3 immunohistochemistry scoring system (Table1). SISH was performed in samples with a IHC score of +2 or +3 . In SISH assessments, HER2 signals and chromosome 17 signals were counted in at least 20 cells, and HER2/CEP17 ratio was calculated. Samples were considered HER2 positive if the HER2/CEP17 ratio calculated by SISH was $\geq 2$. If the IHC score was 0 or +1 , the tumor was considered HER2 negative, and SISH was not performed in these samples.

\section{Statistical Analysis:}

A GraphPad Prism software package 5.00 was used for statistical analyses. A p-value less than 0.05 was considered statistically significant. The Shapiro-Wilk test was used to determine if the data were normally distributed. Oneway variance analysis was used to compare means of more than two groups, and two-way ANOVA analysis was used to compare two independent groups.

\section{RESULTS}

$84(70 \%)$ out of 120 patients with metastatic gastric carcinoma were male, and $36(30 \%)$ were female, and the mean age was 60.7 years (age range: 21-90 years). 84 patients had undergone an endoscopic biopsy, and 36 patients had undergone gastric resection, whereas 39 patients were diagnosed in other health facilities and were referred to our department for consulting.

The most common site of metastasis was the liver (33\%), followed by the esophagus $(9.4 \%)$. Other sites of metastases are shown in Table 2.

Preparations were assessed in terms of HER2 overexpression 54 (45\%) subjects tested negative for HER2 
expression (IHC $0,+1), 48(40 \%)$ patients were suspicious for HER2 overexpression (IHC +2) and $18(15 \%)$ tested positive for HER2 expression (IHC +3). SISH was negative in samples from 37 (77) subjects who scored +2 in ICH and SISH was positive in samples from 11 (23\%) subjects (Figure1). HER2 amplification was detected by SISH in all of 20 subjects $(100 \%)$ who scored +3 in IHC (Figure 2$)$.

In total 31(25.8\%) out of 120 subjects tested positive for HER2 overexpression (Table 3).

The tumor was located in the gastric antrum in 55 subjects (46\%), in the cardia in 29 subjects (24.2), in the gastric fundus in 20 subjects (16.6\%), and cancer involved the entire stomach in 16 subjects (13.3\%) (Table 4).

Based on WHO classification, the histologic pattern of gastric carcinoma was tubular in 82 subjects $(68.3 \%)$, signet-ring cell pattern in $18(15 \%)$, mucinous in 6 subjects $(5 \%)$, a mixed pattern in 13 subjects $(10.8 \%)$ and papillary in 1 subjects (Table 4 ).

Based on Lauren's classification, 89 subjects (74\%) had intestinal-type carcinoma, 18 subjects (15\%) had diffuse-type carcinoma, and 13 subjects $(10.8 \%)$ had mixed type carcinoma (Tablo4).

Among 36 subjects who had undergone gastric resection vascular invasion was detected in 23 subjects (63.8\%), lymphatic invasion was detected in 27 subjects $(75 \%)$ and perineural invasion was detected in 23 subjects $(63.8 \%)$. Regional lymph node involvement was observed in 33 subjects (\%91) (Table 4)

The assessment of clinical and clinicopathological characteristics of HER2 positive subjects revealed that 24 subjects were male $(77.4 \%)$ and 7 subjects were female $(22.5 \%)$. The mean age of HER2-positive study subjects was 62.8 years.

Among subjects with liver metastases, 11 (28.94\%) had HER2 positive tumors, and among subjects with esophageal metastases 7 (63.6\%) subjects HER2 positive tumors (Table 2). Among subjects with HER2 positive gastric carcinoma, the tumor was located in the cardia in 16 subjects $(51.6 \%) .29(93.5 \%)$ subjects had intestinal type carcinoma based on Lauren's classification, and none of the subjects with diffuse-type carcinoma tested positive for HER2 overexpression. The mean tumor diameter was $5.8 \mathrm{~cm}$ (range: $2.5-9 \mathrm{~cm}$ ). Angio-invasion was detected in seven subjects $(22.5 \%)$, lymphatic invasion was detected in 8 subjects $(25.8 \%)$, and perineural invasion was detected in 4subjects (17.4\%) (Table 5).

No statistically significant differences were found between HER2 positive subjects and HER2 negative subjects in age, sex, tumor diameter, T stage, and lymph node metastases. The analysis of tumor location revealed that HER2 positive tumors were more likely to occur in the cardia, whereas HER2 negative tumors were more common in the gastric antrum, and a significant difference was found between the two groups in tumor locations $(\mathrm{p}=0.03)$. (Figure $3 \mathrm{a})$

Comparisons of histological patterns, according to Lauren's classification, indicated that intestinal type was predominant in both groups, and none of the diffuse gastric carcinomas was HER2 positive. A statistically significant intergroup difference was found with respect to the prevalence of diffuse gastric carcinoma $(\mathrm{p}=0.03)$. (Figure 3b)

Comparisons of histological types based on WHO classification revealed that 93 subjects $(77.5 \%)$ had tubular gastric carcinoma, of which $21(22.58 \%)$ tested positive for HER2 overexpression. No significant difference was found between the two groups. Tubular tumors were poorly differentiated in both groups (Figure $3 \mathrm{c}$ ).

No statistically significant differences were found between HER2 positive and HER2 negative groups in angioinvasion, lymphatic invasion, and perineural invasion.

All tumors were considered to be stage 4 (T4) as distant metastases were found in all subjects. Lymph node involvement was detected in 27 subjects (75\%). Lymph node involvement was less common in HER2-positive subjects $(16.6 \%)$ compared to HER2 negative subjects $(77.7 \%)$. The difference was not considered statistically significant.

\section{DISCUSSION}

In many patients, gastric carcinoma is diagnosed at advanced and metastatic stage. According to the International Agency for Research on Cancer (IARC), 951,000 new diagnoses of gastric carcinoma were made worldwide in 2012, and 723.000 of these cases resulted in death. Gastric carcinoma ranks fifth among the most common cancers and ranks third among the most common causes of cancer death worldwide. More than $70 \%$ of all cases of gastric carcinoma are diagnosed in developed countries (12).

Overexpression of HER2 oncoprotein in gastric carcinoma was first defined in 1986. Various studies have demonstrated that oncoproteins might play a role in the development of gastric carcinoma (9).

The addition of anti-HER2 (Human epidermal growth factor-2) monoclonal antibody Trastuzumab to chemotherapy has been demonstrated to improve survival in studies aiming to develop new treatment modalities as an adjuvant to chemotherapeutic agents.

HER2 gene amplification has been reported to occur in gastric carcinoma at rates varying between $7 \%$ and $34 \%$. This variability may occur as a result of various factors, including sample size, tumor heterogeneity, differences in patient populations, and differences in methods or scoring systems used in the study (6).

HER2 expression rate was found to be $22.1 \%$ in the ToGA study investigating the basis for the use of anti- 
HER2 therapy in gastric carcinoma in 3807 patients with gastric carcinoma from 24 countries, HER2 expression rate was found to be $22.1 \%$ (6). HER2 expression rate was found to be $29.5 \%$ by Ljuubomir Ognjenovic et al.(12) and $8 \%$ by Tevfik et al. (13). In line with the literature, the prevalence of HER2 gene amplification in gastric carcinoma was found to be $25.8 \%$ in this study.

Gastric carcinoma is more likely to occur over the age of 60 years and in males, and no significant differences have been observed between patients with HER2 positive gastric carcinoma and patients with HER2 negative gastric carcinoma regarding age and gender predilection $(7,13,14)$. In this study, $70 \%$ of study subjects were males with a mean age of 62.8 years.

Many recent publications are reporting that gastric carcinomas are more likely to occur in the proximal stomach $(9,15,16)$ Isabel A et al. reported that tumors were more common in the proximal stomach in a publication on HER2 expression investigated in 93 patients with gastric carcinoma (9). The prevalence of HER2 expression was found to be $34 \%$ in tumors located in the proximal stomach and $20 \%$ in tumors located in the distal stomach in the ToGA study, which included patients with gastric carcinoma from 122 sites in 24 countries (6). In our study, $44.4 \%$ of HER 2 positive tumors were located in the proximal stomach, whereas $84.4 \%$ of HER2-negative tumors were located in the distal stomach in line with the literature, and a statistically significant intergroup difference was detected in tumor locations $(\mathrm{p}=0.016)$. (Table 4$)$

Several studies have reported an association between HER2 overexpression and poor prognosis $(8,9,10,11)$, whereas no statistically significant association was found between HER2 overexpression and poor prognosis in many studies $(13,14,15,16,17,18,19)$.

In a meta-analysis of 41 studies including 17.494 subjects Yu-Ying Lei et al. determined that there was no statistically significant association between a positive HER2 overexpression and age, tumor size, lymphovascular and neural invasion. In contrast, statistically significant associations were found between HER2 overexpression and sex, tumor locations, TNM stage system, distant metastasis, lymph node metastasis, Lauren's classification, and the degree of tumor differentiation. In that study investigators concluded that tumors tested positive for HER2 were associated with poor prognosis (11). In our study gastric carcinomas were of intestinal type by Lauren's classification and none of the cases of diffuse gastric carcinoma tested positive for HER2. Therefore, a statistically significant intergroup difference was found in histological types by Lauren's classification ( $p=0.03$ ) (Table 4).

Meltem B et al. assessed potential associations between HER2 status by immunohistochemistry and FISH (Fluorescence in situ hybridization) and clinical and pathological parameters and prognosis in 598 subjects with gastroesophageal carcinoma. The rate of HER2 positive tumors was found to be $15 \%$, and unlike other studies in the literature, no significant differences were found between proximal and distal locations of the tumor. HER2 overexpression was more likely to be significant in well-differentiated tumors and tumors of intestinal-type compared to poorly differentiated tumors, and no significant associations were found between HER 2 overexpression and other prognostic factors (17). In our study, all subjects had poorly differentiated tumors and no statistically significant differences were found between HER2 positive and HER2 negative subjects in terms of lymphatic, perineural, and vascular invasion (Table 5).

Tevfik K. et al. investigated the prevalence of HER2 overexpression and clinical parameters and prognosis. They determined that $52 \%$ of tumors were located in the distal stomach and HER2 overexpression had no significant effects on overall and disease-free survival. (13)

In several studies, HER2 overexpression was observed in well-differentiated and moderately differentiated gastric carcinoma tumors $(7,13,14,15,16,19)$, whereas poorly differentiated tumors were found to overexpress HER2 in other studies $(8,10,11,18)$. Tumors overexpressing HER2 in our study were poorly differentiated and no significant difference was found between HER2 positive and HER2 negative subjects in tumor differentiation.

Chao He et al. reported higher rates of HER2 positivity $(26.47 \%)$ in poorly differentiated gastric carcinoma without lymph node metastasis, although many studies have reported numerically higher rates of lymph node metastasis in subjects with HER2 positive tumors (7). In our study, the prevalence of lymph node metastasis was higher in subjects with HER2- negative tumors (77.7\%) compared to those with HER2-positive tumors.

W.Q Sheng et al. investigated clinicopathological characteristics and HER2 status in 726 subjets with gastric cancer from 4 different clinics representative of four different geographic regions of China. The prevalence of HER2 positivity correlated with sex, tumor location, histological grade, and Lauren's classification and HER positivity was more common in gastric carcinoma of intestinal type, well and moderately differentiated tumors, gastroesophageal carcinomas and in males. No statistically significant associations were found between HER2positive status and advanced age, lymph node metastasis, $\mathrm{pT}$ stage, $\mathrm{pN}$ stage or $\mathrm{pM}$ stages (16).

Although conflicting results have been reported in the literature, it is remarkable that HER2 positive tumors are of Lauren's intestinal morphology, are moderately differentiated or well-differentiated, are located in the proximal stomach, and associated with lymph node metastasis and advanced pT stage $(15,16)$. In our study, tumors overexpressing HER2 were poorly differentiated, mostly were of intestinal morphology and located in the cardia. HER2 overexpression was not detected in any cases of diffuse stomach carcinoma. However, no statistically significant associations were found between HER2 overexpression and histopathological parameters. These 
differences among studies might be associated with geographic characteristics, sample size, study methods, and also suggest that HER2 might be a prognostic factor in stomach carcinoma.

This study was performed to determine the prevalence of HER2 overexpression, which might show geographic variations, to perform a comparative analysis of HER2 expression by immunohistochemistry (ICH) and silver in situ hybridization and to reveal potential associations between HER2 overexpression and clinicopathological parameters.

HER2 positive status has been comprehensively investigated in breast cancer and it has long been considered to be a poor prognostic factor. Trastuzumab is known to improve survival in patients with primary or metastatic HER2 positive breast cancer $(6,10,13)$. Besides, many studies demonstrated that the addition of Trastuzumab to chemotherapy might improve survival in advanced stomach cancers and gastroesophageal junction cancers, and further studies on various pathways may be guiding.

Table1: IHC scoring criteria for HER2

\begin{tabular}{|l|l|l|}
\hline Score & Staining Pattern & HER2 status \\
\hline 0 & No staining & Negative \\
\hline $1+$ & In $>10 \%$ of cells, membranous, faint & Negative \\
\hline $2+$ & In $>10 \%$ of cells, membranous, moderate, basolateral/lateral & Indefinite \\
\hline $3+$ & In $>10 \%$ of cells, membranous intense, basolateral/lateral & Positive \\
\hline
\end{tabular}

Table 2: Sites of metastasis and their frequency in gastric carcinoma

\begin{tabular}{|l|l|l|l|}
\hline Site of Metastasis & $\begin{array}{l}\text { Number } \\
(\%)\end{array}$ & HER2 $(+)(\%)$ & $\begin{array}{l}\text { HER2 } \\
(-)(\%)\end{array}$ \\
\hline Liver & $38(32.47)$ & $11(28.4)$ & $25(65.78)$ \\
\hline Esophagus & $11(9.4)$ & $7(63.6)$ & $4(36.36)$ \\
\hline Omentum & $10(8.54)$ & $3(30)$ & $7(70)$ \\
\hline Bone & $9(7.69)$ & $3(33.33)$ & $6(66.67)$ \\
\hline Lung & $9(7.69)$ & $2(22.22)$ & $7(77.78)$ \\
\hline Colon & $6(5.12)$ & - & $6(100)$ \\
\hline Ovary & $4(3.41)$ & - & $4(100)$ \\
\hline Pleura & $4(3.41)$ & $2(50)$ & $2(50)$ \\
\hline Breast & $1(0.85)$ & $1(100)$ & \\
\hline Peritoneum & $4(3.41)$ & & \\
\hline Pancreas & $2(1.71)$ & $2(100)$ & $2(100)$ \\
\hline Soft Tissue & $2(1.71)$ & - & \\
\hline
\end{tabular}

Table 3: HER2 Analysis in gastric tumor tissues:

\begin{tabular}{|l|c|c|c|c|}
\hline & IHC $0 / 1+$ & IHC $2+$ & IHC 3+ & Total N (\%) \\
\hline SISH positive & 0 & $11(23 \%)$ & $20(100 \%)$ & $31(25.8)$ \\
\hline SISH negative & $52(100 \%)$ & $37(77 \%)$ & 0 & $89(75 \%)$ \\
\hline Total & $52(43.3 \%)$ & $48(40 \%)$ & $20(16.6 \%)$ & 120 \\
\hline
\end{tabular}


Table 4: Comparisons between HER2 positive and HER2-negative subjects in clinical and pathological parameters

\begin{tabular}{|c|c|c|c|c|}
\hline & $\begin{array}{l}\text { Total } \\
(\mathrm{n}=120)(\%)\end{array}$ & $\begin{array}{l}\text { HER2 }(+) \\
(\mathrm{n}=31) \\
(\%)\end{array}$ & $\begin{array}{l}\text { HER2(-) } \\
(\mathrm{n}=89) \\
(\%) \\
\end{array}$ & $\mathrm{P}$ value \\
\hline Age (mean) & 60.7 & 62.8 & 60.1 & \\
\hline \multicolumn{5}{|l|}{ Sex } \\
\hline Male & $84(70)$ & & & \\
\hline Female & $36(30)$ & & & \\
\hline Tumor location & & & & 0,030 \\
\hline Antrum & $58(48.33)$ & $9(15.51)$ & $49(84.48)$ & \\
\hline Body & $19(15.83)$ & $5(26.31)$ & $14(73.68)$ & \\
\hline Cardia & $36(30)$ & $16(44.44)$ & $20(55.56)$ & \\
\hline Entire stomach & $7(5.83)$ & $1(14.28)$ & $6(85.71)$ & \\
\hline Lauren's classification & & & & 0,03 \\
\hline Intestinal & $97(80.83)$ & $29(29.89)$ & $68(70.10)$ & \\
\hline Diffuse-mixed & $19-4(19.16)$ & $2(8.69)$ & $21(91.30)$ & \\
\hline WHO classification & & & & 0,32 \\
\hline Tubular & $93(77.5)$ & $21(22.58)$ & $72(77.41)$ & \\
\hline Well-differentiated & $3(3.22)$ & $1(33.33)$ & $2(66.67)$ & \\
\hline Moderately-differentiated & $38(40.86)$ & $13(34.21)$ & $17(44.73)$ & \\
\hline Poorly-differentiated & $52(55.91)$ & $17(32.69)$ & $45(86.53)$ & \\
\hline Papillary & $1(0.83)$ & 1 & - & \\
\hline Mucinous & $4(3.33)$ & $1(25)$ & $3(75)$ & \\
\hline Signet ring cell-Poor cohesive carcinoma & $19(15.83)$ & $1(5.26)$ & $18(94.73)$ & \\
\hline Mixed & $3(2.5)$ & $1(33.33)$ & $2(66.67)$ & \\
\hline Tumor size & $7.5 \mathrm{~cm}(17.5-2.5)$ & $5.8 \mathrm{~cm}(9-2.5)$ & $8.4 \mathrm{~cm}(15.5-2.5)$ & 0.11 \\
\hline
\end{tabular}

Table 5: Comparisons clinical and pathological parameters between HER2 positive and HER2-negative subjects who undergone gastric resection

\begin{tabular}{|l|l|l|l|l|}
\hline & Total resection $(\mathrm{n}=36)(\%)$ & $\begin{array}{l}\text { HER2 }(+) \\
(\mathrm{n}=10)(\%)\end{array}$ & $\begin{array}{l}\text { HER2(-) } \\
(\mathrm{n}=26)(\%)\end{array}$ & P value \\
\hline Vascular invasion & & & & 0.71 \\
\hline Positive & $23(63.88)$ & $7(30.4)$ & $16(69.6)$ & \\
\hline Negative & $13(36.11)$ & $3(23)$ & $10(77)$ & \\
\hline Perineural invasion & & & & 0.11 \\
\hline Positive & $23(63.88)$ & $4(17.4)$ & $19(82.6)$ & \\
\hline Negative & $13(36.11)$ & $6(46.1)$ & $7(53.9)$ & \\
\hline Lymphatic invasion & & & & 1 \\
\hline Positive & $27(75)$ & $8(29.6)$ & $19(70.3)$ & \\
\hline Negative & $9(25)$ & $2(22.3)$ & $7(77.7)$ & \\
\hline & & & & 0.32 \\
\hline Lymph node metastasis & $27(75 \%)$ & $6(22.3 \%)$ & $21(77.7 \%)$ & \\
\cline { 1 - 3 }
\end{tabular}




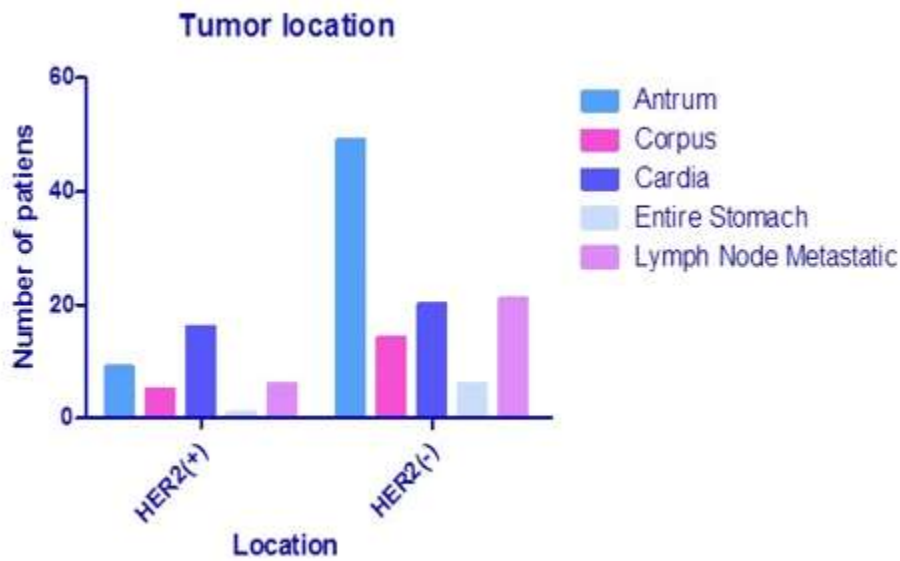

FIGURE3a:.HER2 status by tumor localization

Lauren classification

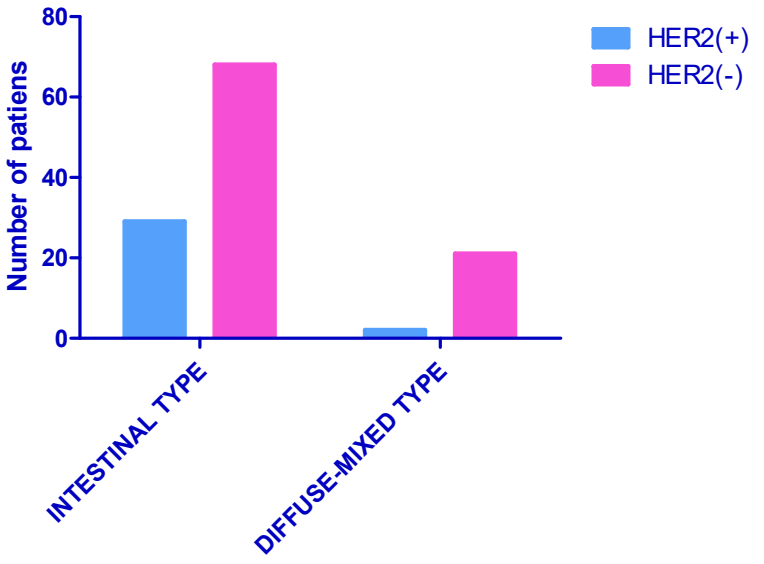

FIGURE3b: HER2 condition in intestinal and diffuse-mixed gastric carcinomas according to Lauren classification

\section{Tubuler type}

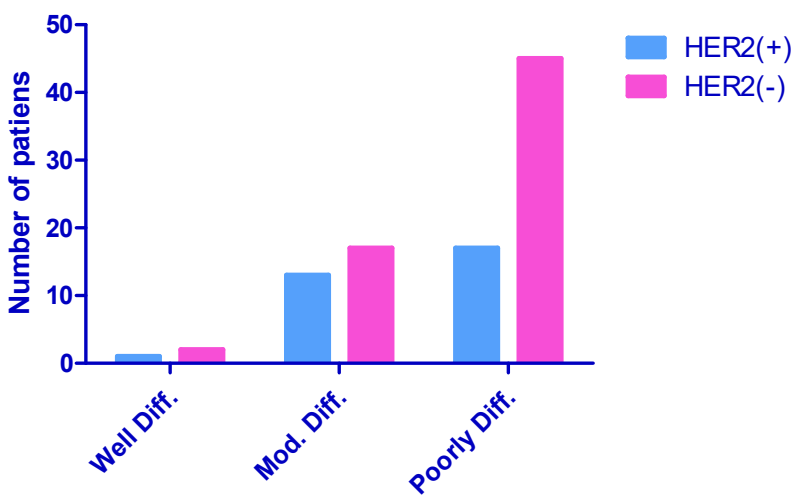

FIGURE3c:HER2 state by degree of differentiation 


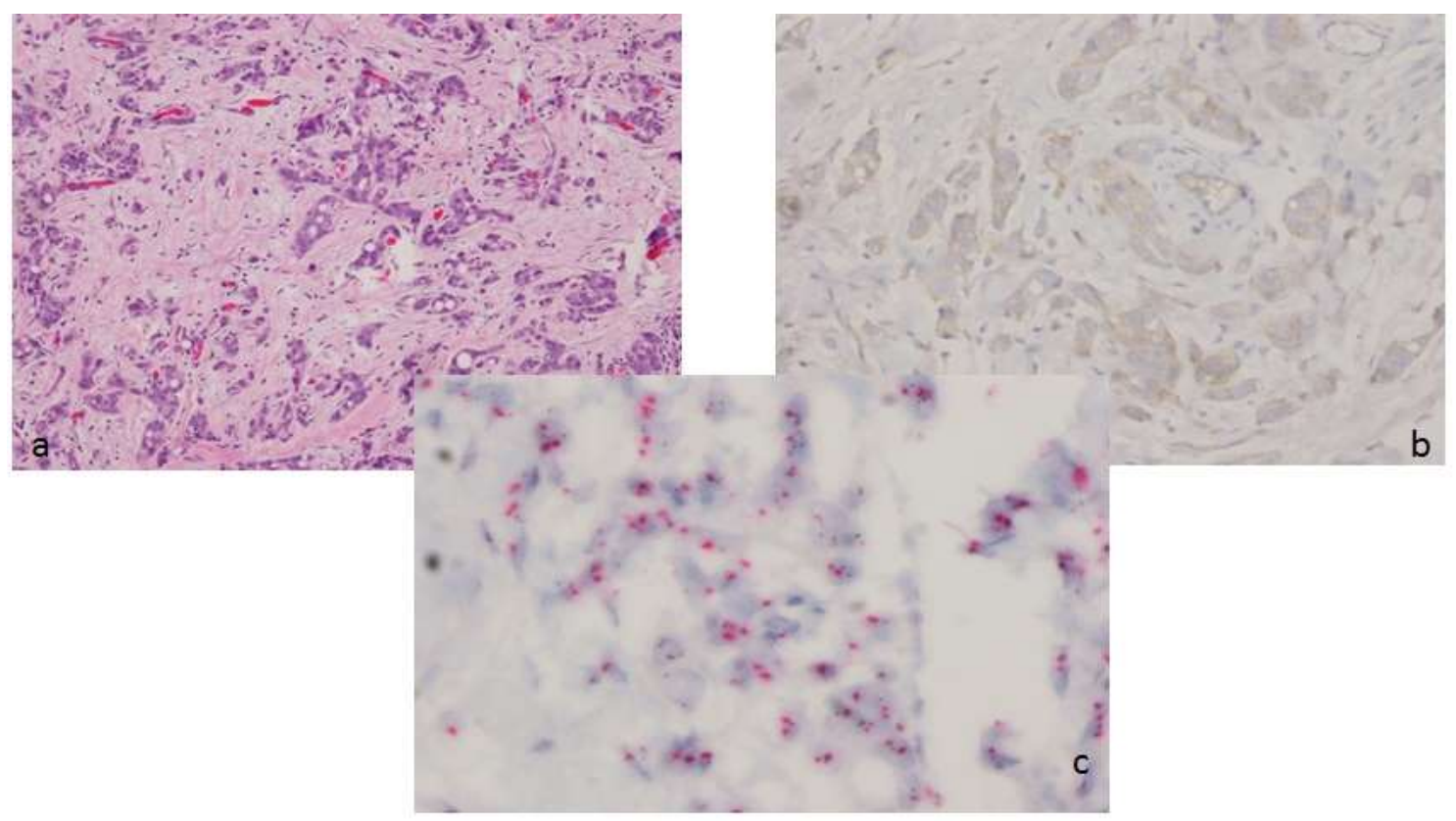

Figure1:Poorly differentiated gastric carcinoma without HER2 protein expression and with gene amplification.a: $\mathrm{H}-\mathrm{E}, \times 200 ; b$ : IHC, HER2 score $2+, \times 200 ; \mathrm{c}$ : SISH (-), $\times 400$

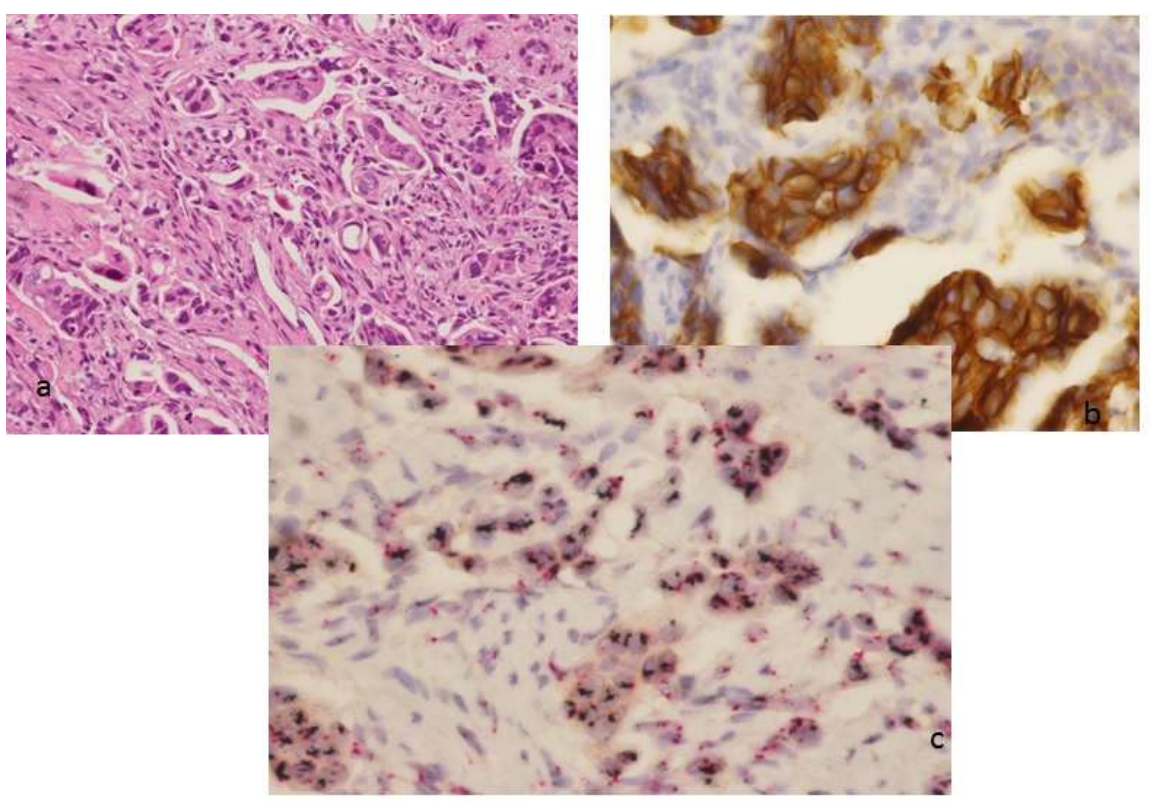

Figure2:Poorly differentiated gastric carcinoma with HER2 protein expression and with gene amplification. a: H-Ex200; b:IHC, HER2 score 3+, $\times 400$; c: SISH (+), $\times 400$. 


\section{Reference}

1-Yalcin S. "Gastric Cancer in Turkey-A Bridge Between West and East". Gastrointestinal Cancer Research : GCR (2009);3 (1): 29-32.

2- Guner . "Recent trends of gastric cancer treatment in Turkey". Translational Gastroenterology and Hepatology 2017:2 https://doi.org/10.21037/tgh.2017.04.01.

3- Hofmann M, Stoss O, Shi D, Assessment of a HER2 scoring system for gastric cancer: results from a validation study. - Abstract - Europe PMC. 2020. https://europepmc.org/article/med/18422971. 4-Irkkan S Ç. "HER2 Assessment in Gastric Carcinoma”. Acta Oncologica Turcica (2014);47 (3): $42-51$. https://doi.org/10.5505/aot.2014.24633.

5-Werner D, Achim B, Kristina S, at all "The validation of a novel method combining both HER2 immunohistochemistry and HER2 dual-colour silver in situ hybridization on one slide for gastric carcinoma testing”. Journal of translational medicine 2014;12: 160. https://doi.org/10.1186/1479-5876-12-160.

6-Bang, Y J, Eric V C, Andrea F, at all. "Trastuzumab in Combination with Chemotherapy versus Chemotherapy Alone for Treatment of HER2-Positive Advanced Gastric or Gastro-Oesophageal Junction Cancer (ToGA): A Phase 3, Open-Label, Randomised Controlled Trial”. The Lancet 2010;376 (9742): 687-97. https://doi.org/10.1016/S0140-6736(10)61121-X.

7-He C, Xue-Yi B, Xing-Zhi N, at all.“Correlation of human epidermal growth factor receptor 2 expression with clinicopathological characteristics and prognosis in gastric cancer". World Journal of Gastroenterology : WJG 2013;19 (14): 2171-78. https://doi.org/10.3748/wjg.v19.i14.2171.

8-Cidon E. U, Centeno R. G, Lagarto E. G,at all. "HER-2 Evaluation in a Specific Gastric Cancer Population with the Highest Rate of Mortality in Spain”. Journal of Oncology $2011 ; 1-6$.

https://doi.org/10.1155/2011/391564.

9-Alvarado C.I, Sara G. H, Ana R. P, at all.“Evaluación por inmunohistoquímica de la expresión del HER2 en cáncer gástrico. Estudio clínico-patológico de 93 casos”. Cirugía y Cirujanos 2017;85 (6): 504-9.

https://doi.org/10.1016/j.circir.2016.11.016.

10-Otsu H, Eiji O, Ayae I.Y,at all. "Correlation of HER2 Expression with Clinicopathological Characteristics and Prognosis in Resectable Gastric Cancer". Anticancer Research, 2015; 35(4): 2441-46.

11-Lei Y, Jin-yu H, Qiong-rui Z, at all. "The clinicopathological parameters and prognostic significance of HER2 expression in gastric cancer patients: a meta-analysis of literature". World Journal of Surgical Oncology 2017; 15 https://doi.org/10.1186/s12957-017-1132-5.

12-Ognjenovic L, Gjorgji T, Stojan G, at all. "HER2 Positive Gastric Carcinomas and Their Clinico-Pathological Characteristics". Open Access Macedonian Journal of Medical Sciences 2018;6 (7): 1187-92.

https://doi.org/10.3889/oamjms.2018.280.

13- Kivilcim Uprak Tevfik, Wafi Attaallah, Cigdem Ataizi Celikel at all. "HER-2 incidence in gastric cancer, its association with prognosis and clinicopathological parameters". Turkish Journal of Surgery .2015;31(4): 207-13. https://doi.org/10.5152/UCD.2015.2964.

14- Shabbir A, Muhammad A Q, Abdullah B K,at all. "Gastric adenocarcinoma expressing human epidermal growth factor receptor in South Asian population”. Saudi Journal of Gastroenterology : Official Journal of the Saudi Gastroenterology Association 2018;24 (5): 289-93.

15--Xieng -S-F, Jie-Yu C, Chang-Feng L, at all ."Differences in HER2 over-expression between proximal and distal gastric cancers in the Chinese population". World Journal of Gastroenterology : WJG 2013;19: 3316-23. https://doi.org/10.3748/wjg.v19.i21.3316.

16-Sheng WQ, D. Huang, J M. Ying, at all. "HER2 Status in Gastric Cancers: A Retrospective Analysis from Four Chinese Representative Clinical Centers and Assessment of Its Prognostic Significance”. Annals of Oncology 2013;24 (9): 2360-64. . https://doi.org/10.1093/annonc/mdt232

17- Meltem B, Mustafa B, Ozgur E, at all. "Clinical Significance of HER2 Overexpression in Gastric and Gastroesophageal Junction Cancers”. Journal of Gastrointestinal Surgery 2015;19 (9): 1565-71.

https://doi.org/10.1007/s11605-015-2888-y.

18-Ozlem T, Serife B, Fevziye K .Mide karsinomlarında C-Erbb-2 (Her-2/Neu) ve P53 Ekspresyonlarının Prognostik Parametrelerle İliskisi. Turkiye Klinikleri J Med Sci 2011;31(3):575-82. doi:10.5336/medsci.200915977

19-Coskun T, Semin A, Ayca T,at all.The relationship between her-2 positivity and histopathological findings in gastric carcinomas. Journal of Current Pathology 2018;32: 85. https://doi.org/10.5146/jcpath.2018.32. 